\begin{tabular}{|c|c|}
\hline Title & Predictive model of cationic surfactant binding to humic substances \\
\hline Author(s) & Ishiguro, Munehide; Koopal, Luuk K. \\
\hline Citation & $\begin{array}{l}\text { Colloids and Surfaces A: Physicochemical and Engineering A spects, 379(1-3), } 70-78 \\
\text { https://doi.org/10.1016/.colsurfa.2010.11.075 }\end{array}$ \\
\hline Issue Date & 2011-04 \\
\hline DOC URL & http:/hdl.handle.net/2115/68458 \\
\hline Rights & $\begin{array}{l}\text { Copyright @ } 2010 \text { Elsevier B.V. A Il rights reserved., @ 2010. This manuscript version is made available under the CC- } \\
\text { BY-NC-ND } 4.0 \text { license }\end{array}$ \\
\hline Rights(URL) & http://creativecommons.org/icenses/by-nc-nd/4.0/ \\
\hline Type & article (author version) \\
\hline File Information & Predictive modelBINDING OF IONIC SURFACTANTSTO HUMIC ACID-1.pdf \\
\hline
\end{tabular}

Instructions for use 


\section{Predictive model of cationic surfactant binding to humic substances}

Munehide Ishiguro $^{*, 1}$, Luuk K. Koopal ${ }^{2}$

${ }^{1}$ Graduate School of Environmental Science, Okayama University, 3-1-1. Tsushima-naka, Kitaku, Okayama 700-8530, Japan

${ }^{2}$ Laboratory of Physical Chemistry and Colloid Science, Wageningen University, Dreijenplein 6, 6703 HB Wageningen, The Netherlands.

* Corresponding author

Tel./fax: +81862518875

E-mail adress: ishi@cc.okayama-u.ac.jp (M. Ishiguro) 


\section{Abstract}

The humic substances (HS) have a high reactivity with other components in the natural environment. An important factor for the reactivity of HS is their negative charge. Cationic surfactants bind strongly to HS by electrostatic and specific interaction. Therefore, a surfactant binding model is developed that takes both the specific and electrostatic interactions explicitly into account. The model is analogous to that of ion binding to HS with the NICA-Donnan model, but competition for the binding sites is not taken into account and the NICA-Donnan model reduces to the Langmuir-Freundlich-Hill-Donnan (LFH-D) model. The parameters of the LFH equation are the maximum binding, the affinity constant and the non-ideality constant. The nonideality parameter accounts for both the site heterogeneity and the cooperativity due to hydrophobic interaction between surfactant molecules. The affinity constant incorporates the specific (e.g., hydrophobic) interactions between surfactant and HS. The Donnan part of the model accounts in a simple way for the electrostatic interactions by assuming that for a given set of conditions there is only one electrostatic potential that governs the behavior in the Donnan phase. The separation between the specific interactions (LHF) and the electrostatic interactions (D) is based on the so-called master curve (MC) procedure in which the binding is replotted as a function of the "free" surfactant concentration in the Donnan Phase. The MC depends only on the specific interactions. Once the MC is obtained it can be fitted to the LFH equation to obtain the model parameters. Subsequently, the surfactant isotherms can be calculated with the LFH-D model. The model is tested using previously obtained data on dodecyl pyridinium chloride (DPC) and cetyl pyridinium chloride (CPC) binding to some purified humic and fulvic acids at $\mathrm{pH}$ about 5. The LFH-D model is well suited to describe the surfactant binding to HAs from very low concentrations up to the iso-electric point (IEP). The affinity of DPC for the different HAs allows ranking of the HAs according to their hydrophobicity. Prediction of DPC binding at other $\mathrm{pH}$ values also leads to good results for HA. For FAs the model can only describe the surfactant 
draft IAP 2010; 3/6/2018

binding up to an adsorbed amount of $0.5 \mathrm{~mol} / \mathrm{kg}$. For higher binding values the LFH-D model underestimates the binding.

\section{Keywords}

NICA model; Donnan model; Langmuir Freundlich equation; Hill equation; surfactant binding model; electrostatic interactions; specific interactions; hydrophobic interactions; humic acid; fulvic acid; dodecyl pyridinium chloride; hexadecyl pyridinium chloride; 


\section{Introduction}

Dissolved humic substances (HS) are organic compounds that commonly exist in soil solutions and natural waters. They can be divided in fulvic acids (FAs) and humic acids (HAs). FAs are soluble in water at all $\mathrm{pH}$ and $\mathrm{HAs}$ are soluble at $\mathrm{pH}>2$. FAs can be considered as oligomeric polyfunctional acids and HAs as structured polyelectrolyte particles with internal chemical and physical links. Due to the presence of a large variety of functional groups HS have a high reactivity with other components in the natural environment and affect the migration of pollutants. An important factor for the reactivity of HS is their negative charge, which is due to the dissociation of the acid groups. Proton binding to HS is well studied; it can be used to characterize HS from physical point of view [1,2] and is the basis for a better understanding of the binding behavior of other ions [3]. Adsorption of heavy metal ions is well documented and both the NICA-Donnan model [4-10] and Model VI [11] are able to describe and predict metal ion binding to $\mathrm{HS}$ as a function of $\mathrm{pH}$, salt concentration and concentration of other competing ions. Also complex formation of HS with cationic polyelectrolytes is well studied. Polyelectrolyte binding to HS can be used to obtain insight in the charge density of the humics $[12,13]$. The main emphasis of these studies is however on the optimization of the complexation conditions for the removal of HS from aqueous solution by selective flocculation [14]. Recently also protein binding to HS has been studied $[15,16]$ and some studies exist on the role that HS have on enzyme activities [17]. HS also can bind low molar mass organic pollutants [18-22], but here the knowledge of the effect of environmental conditions such as $\mathrm{pH}$ and salt concentration on the binding is mainly limited to surfactant binding.

Surfactants are an important class of low molar mass components. Surfactants are widely used at home and in industry; they are the most discharged synthetic chemicals to the environment and they may destroy cell membranes of living organisms [23]. Surfactant binding to mineral oxides has been studied over decades and sophisticated 
draft IAP 2010; 3/6/2018

binding models exist [24-28]. Direct studies of surfactant binding to HS are limited and more recent [21, 22, 29, 30]. Koopal et al. [29] have shown the influence of $\mathrm{pH}$ and cationic surfactant aliphatic chain length on the binding of cationic surfactants to HA. In a similar study Yee et al. [21] have measured cationic surfactant binding to HA and FA and the results were analyzed using the Hill and the Langmuir equation. The surfactant binding to FA could be described with the Hill equation; it showed, close to the critical micelle concentration of the surfactants, a strong cooperativity that was not present with the binding to HA. The surfactant binding to HA could be described with the Langmuir equation. Ishiguro et al. [22] have measured alkyl-pyridinium surfactant isotherms to HAs and FAs over a large surfactant concentration range and investigated the effects of salt concentration and surfactant chain length on the binding. In a recent study Matsuda et al. [30] investigated the effects of chain length, salt ion concentration and the presence of heavy metal cations on the binding of cationic surfactants to HA. Although some modeling attempts have been made [21], unraveling of the electrostatic and specific interactions that contribute to the surfactant binding to HS, as done for ion binding, has not yet been considered. Only by considering both types of interactions explicitly models based on intrinsic binding parameters can be obtained that allow the prediction of the surfactant binding to $\mathrm{HA}$ and FA as a function of surfactant concentration, $\mathrm{pH}$ and salt concentration. Therefore, in this paper a modeling route for surfactants has been chosen that is closely related to the NICA-Donnan model $[9,10]$ used for proton and metal ion binding to HS. The electrostatic interaction is modeled using the Donnan model that allows the calculation of the electrostatic potential near the sites. The specific interactions are taken into account by using a simplified form of the NICA (non-ideal competitive adsorption) equation. When the surfactant adsorption occurs mainly on the dissociated 
draft IAP 2010; 3/6/2018

acidic groups the site completion can be neglected and the NICA model can be simplified to the Langmuir-Freundlich-Hill (LFH) model for mono-component non-ideal adsorption. In the LFH model non-idealities due to heterogeneity and cooperativity (hydrophobic interaction) are included in one non-ideality parameter. When the full NICA model would have been used a separation between heterogeneity and cooperativity would be required. Separation of both interactions is difficult and it would make the parameter interpretation unnecessary complicated. By neglecting site competition there is no direct competition between surfactant cations and protons, but adaptation of proton binding as a result of the changing electrostatic interactions caused by the surfactant binding can easily be incorporated in the model.

In the paper first the model will be explained in detail. Then the binding data of hexadecyl (=cetyl) pyridinium chloride (CPC) and dodecyl pyridinium chloride (DPC) to purified Aldrich humic acid (PAHA), Dando humic acid (DHA), Inogashira humic acid (IHA), Laurentian fulvic acid (LFA) and Strichen fulvic acid (SFA) [22] will be used to test the model. For PAHA a prediction will be made of the $\mathrm{pH}$ dependence of the binding and this prediction will be compared with the results obtained by Koopal et al. [29]. A brief discussion will be given of the obtained parameter values.

\section{Surfactant Binding Model}

\subsection{General considerations and the master curve}

Preferably the description of surfactant binding to humic substances (HS) is done with thermodynamic constants that do not depend on the environmental conditions. To obtain a model that is not too complicated, simplifying assumptions have to be made regarding structure, shape and size of the humic particles and on the way in which the electrostatic and specific interactions will be separated. The assumptions with respect to structure, size and shape of the particles are 
draft IAP 2010; 3/6/2018

especially important for handling the electrostatics; it will be assumed that in aqueous solution HS particles are permeable for ions, monodisperse and spherical. In order to make the separation between electrostatic and specific interactions easy the further assumption is made that the electrostatic potential at the location of the binding sites at given conditions can be represented by one averaged, smeared-out, potential. With these assumptions one neglects: (1) the effects of HS site heterogeneity on the electrostatic potential, (2) the dispersion of sizes and shapes of the HS particles and (3) a potential distribution inside the HS molecule. Consequently, the site potential depends only on the specific $\mathrm{HS}$, the $\mathrm{pH}$, the ionic strength and the amounts of specifically bound ions, but not on the particle details or the detailed location of the functional groups.

Due to the negative electrostatic potential of a HS particle the concentration/activity of an ion in the close vicinity of the functional groups of the particle differs from that in the bulk solution. The apparent affinity of the ion to the binding sites at given conditions consists of an ion-specific (intrinsic) contribution and a generic electrostatic contribution that is related to the site potential. In general, the electrostatic site potential cannot be measured directly; however, the potential can be estimated with the help of an electrostatic model that describes the electrostatic potential as a function of the charge of a HS at the given conditions. Once the electrostatic model is chosen, the site potential can be found. In the case of a mean field approach the ion concentration/activity in solution close to a binding site, $c_{i, \text { loc }}$, can be calculated according to the Boltzmann law:

$$
c_{i, \mathrm{loc}}=c_{i} \exp \left(\frac{-\tau_{i} F \psi_{\mathrm{loc}}}{R T}\right)
$$

where $c_{i}$ is the concentration/activity of ion $i$ in bulk solution, $\tau_{i}$ the valence of ion $i, F$ the Faraday constant, $\psi_{\text {loc }}$ the smeared-out electrostatic potential at the location of the binding sites of the HS particle, $R$ the gas constant and $T$ the absolute temperature.

A set of surfactant isotherms measured at different salt concentrations will in principle reflect the electrostatic interactions and how these are screened. Such data are therefore well suited for testing the electrostatic model. With the electrostatic model $\psi_{\text {loc }}$ can be calculated and therefore 
draft IAP 2010; 3/6/2018

also $c_{i, \text { loc }}$ (see eq. 1) corresponding with $c_{i}$ at a given adsorbed amount can be obtained. Once the $c_{i, \text { loc }}$ values are known the adsorption values can be re-plotted versus the local concentrations. The electrostatic model is adequate when the isotherms at different salt levels merge into a single master curve (MC) when they are plotted vs. this local ion concentration/activity. This procedure has been applied before for proton binding to HS [1,9], but it can be used equally well for surfactant ions. Within the given set of model constraints the MC of surfactant binding is not dependent on the electrostatics and reflects the intrinsic behavior of the binding to the HS. Once an electrostatic model is established it can also be applied to other conditions provided that the contribution of the adsorbing surfactant ions and protons to the charge of the HS is accounted for. Therefore, the electrostatic model can be combined with a specific ion-binding model such as the NICA model that is specifically developed for non-ideal competitive ion binding to HS $[9,10]$. When the competition for the sites is neglected the NICA equation reduces to the LangmuirFreundlich-Hill equation, which can describe the non-ideal binding of one component on a continuous distribution of heterogeneous binding sites [4].

\subsection{Electrostatic model.}

In the case of ion binding to HS several electrostatic models have been proposed [3138]. The most common approach today is to consider an HS particle as a pseudo phase into which water and ions can penetrate. The simplest form of this model is the Donnan model $[37,38,39]$. The mean field smeared-out potential inside the Donnan particle volume is called the Donnan potential $\left(\psi_{\mathrm{D}}\right)$ and eq. (1) applies with $\psi_{\mathrm{loc}}=\psi_{\mathrm{D}}$ and $c_{i, \mathrm{loc}}=$ $c_{i, \mathrm{D}}$, the concentration of ion $i$ within the Donnan particle volume. Outside the Donnan particle volume the electrostatic potential drops to zero. 
The primary volume charge density in the Donnan phase, $\rho_{\mathrm{D}}$, is determined by the proton desorption (dissociation), $X_{\mathrm{H}}$ (mol negative charges/kg), and the cationic surfactant binding, $X_{\mathrm{S}}$ (mol positive charges $\left./ \mathrm{kg}\right)$ :

$$
\rho_{\mathrm{D}}=\frac{F\left(-X_{\mathrm{H}}+X_{\mathrm{S}}\right)}{V_{\mathrm{D}}}
$$

where, $V_{\mathrm{D}}$ is the specific volume of the Donnan phase $\left(\mathrm{m}^{3} / \mathrm{kg}\right)$ and $\rho_{\mathrm{D}}$ is expressed in $\mathrm{C} / \mathrm{m}^{3}$. The specific volume of the Donnan phase can be transformed in the Donnan particle volume when the molar mass of the particles is known. Due to the surfactant binding the electrostatic interaction changes and this affects the proton charge. The proton charge is made up of the proton charge in the absence of surfactant binding plus a proton charge increment due to surfactant binding. When it will be assumed that the negative proton charge increases linearly with the surfactant binding the proton charge can be expressed as:

$$
X_{\mathrm{H}}=X_{\mathrm{H}, 0}+\Delta^{\mathrm{m}} X_{\mathrm{H}} \theta_{\mathrm{S}}
$$

with $X_{\mathrm{H}, 0}$ the proton $(\mathrm{H})$ charge in the absence of surfactant binding, $\Delta^{\mathrm{m}} X_{\mathrm{H}}$ the maximum increment of the proton charge due to surfactant binding, $\theta_{\mathrm{S}}=X_{\mathrm{S}} / X^{\mathrm{m}}$ the degree of surfactant $(\mathrm{S})$ binding and $X^{\mathrm{m}}$ the maximum surfactant binding which equals the possible total charge amount between very low and very high $\mathrm{pH}$.

The specific Donnan volume $V_{\mathrm{D}}$, can be theoretically motivated on the basis of the HS particle size and the Debye length [40] or empirically adjusted. Here Benedetti et al. [33] and Milne et al. [7] will be followed, who applied an empirical equation for $V_{\mathrm{D}}$ that depends on the ionic strength, $I$, and for which the Donnan particle volume is, in general, larger than the particle size:

$$
\log V_{\mathrm{D}}=b(1-\log I)-1 \quad\left(V_{\mathrm{D}} \text { in } \mathrm{L} / \mathrm{kg} ; I \text { in } \mathrm{mol} / \mathrm{L}\right)
$$


draft IAP 2010; 3/6/2018

where $b$ is a constant depending on the specific HS. A practical advantage of this expression is that the molar mass of the particles is not required for the calculation of $V_{\mathrm{D}}$. The concentrations of small ions inside the Donnan phase can be obtained from the condition of electroneutrality in this phase: $\rho_{\mathrm{D}}=-\rho_{\mathrm{d}, \mathrm{D}}$ where $\rho_{\mathrm{d}, \mathrm{D}}$ is the "diffuse" charge in the Donnan phase. When all ions present have the same valence $\tau$ or when a $(\tau-\tau)$ symmetrical background electrolyte determines the ionic strength in solution one obtains

$$
-\rho_{\mathrm{D}}=\rho_{\mathrm{d}, \mathrm{D}}=F \tau\left(c_{+, \mathrm{D}}-c_{-, \mathrm{D}}\right)
$$

where $c_{+, \mathrm{D}}$ is the (total) cation and $c_{-, \mathrm{D}}$ the (total) anion concentration in the Donnan phase. Substitution of the expressions (1) for $c_{i, \mathrm{D}}$ in eq. (5) leads to the expression for $\rho_{\mathrm{D}}$ as a function of $\psi_{\mathrm{D}}$ :

$$
\rho_{\mathrm{D}}=2 \tau c_{\mathrm{s}} F \sinh \left(\frac{\tau F \psi_{\mathrm{D}}}{R T}\right)
$$

By inversion of this relation $\psi_{\mathrm{D}}$ as a function of $\rho_{\mathrm{D}}$ is obtained:

$$
\psi_{\mathrm{D}}=\frac{R T}{\tau F} \operatorname{arcsinh}\left(\frac{\rho_{\mathrm{D}}}{2 \tau c_{\mathrm{s}} F}\right)
$$

where $c_{s}$ is the total salt concentration $\left(\mathrm{mol} / \mathrm{m}^{3}\right)$. Eq. (7) can be used to calculate the Donnan potential when the Donnan charge is known.

\subsection{Langmuir-Freundlich-Hill (LFH) equation}

The NICA equation for surfactant binding to the acid sites of a HS can be written as

$$
X_{a}=X^{m} \frac{\left(K_{S}^{*} \odot_{S}^{*}\right)^{n}}{1+\left(K_{H}^{*} c_{H}^{*}\right)^{2}+\left(R_{S}^{*} c_{S}^{*}\right)^{\mathrm{N}}}
$$

where $X_{\mathrm{S}}(\mathrm{mol} / \mathrm{kg})$ is the bound amount of surfactant, $c_{i}{ }^{*}(\mathrm{~mol} / \mathrm{L} /[1 \mathrm{~mol} / \mathrm{L}])$ the 
draft IAP 2010; 3/6/2018

equilibrium concentration/activity in solution (surfactant, indicated with subscript $i=S$, or proton, indicated with subscript $i=H$ ) normalized with respect to the standard state concentration of $1 \mathrm{~mol} / \mathrm{L}, X^{m}$ the maximum amount of surfactant that can bind $(\mathrm{mol} / \mathrm{kg})$, $K_{i}^{*}$ the intrinsic affinity constant (standard state concentration $1 \mathrm{~mol} / \mathrm{L}$ ) that expresses the intrinsic affinity between a surfactant molecule or a proton and a surface site and $n$ and $p$ are non-ideality parameters of, respectively, the surfactant and the proton, which account for the fact that the (actual) affinity of the ions for the binding sites varies with surface coverage. When $1+\left(K_{H}^{8} c_{H}^{8}\right)^{\mathrm{p}} \approx 1$, that is at not too low $\mathrm{pH}$, the proton is relatively unimportant and the NICA equation reduces to

$$
X_{g}=X^{m} \frac{\left(K_{S}^{s} c_{S}^{s}\right)^{n}}{1+\left(K_{S}^{*} c_{S}^{8}\right)^{n}}
$$

which can be interpreted as the mono-component version of the NICA equation or the LFH equation.

When the binding is ideal, the affinity does not depend on the concentration or the binding and $n=1$; eq. (9) reduces to the Langmuir equation. When the binding shows a positive cooperativity the affinity increases with increasing coverage and/or concentration and $n>1$. In this case eq. (9) is known as the Hill equation [41, 42]. When the binding occurs on sites that are heterogeneous, the affinity gradually decreases because the sites with high affinities will be preferentially occupied at low concentration and the low affinity sites will be occupied at high concentration and $n<1$. In this case $K^{*}\left(=K^{*} S\right)$ is the affinity corresponding with the peak of the distribution function of $\log K^{\prime}$ and eq. (9) is the so-called Langmuir-Freundlich equation [43,44]. A value of $n<1$ can also be regarded as negative cooperativity due to a stoichiometry in which one adsorbate molecule occupies more than one binding site, but such behavior is not relevant for surfactants. For surfactant binding to HS both the heterogeneity of the HS binding sites 
draft IAP 2010; 3/6/2018

and a positive cooperativity due to hydrophobic attraction will play a role. To express this in the name of eq. (9) it is called the Langmuir-Freundlich-Hill equation and $n$ can be smaller or larger than unity. The LFH eq. with $n<1$ implies that the heterogeneity effects are larger than the hydrophobic effects and $n>1$ implies that the hydrophobic attraction is stronger than the heterogeneity effect. Pseudo-ideality $(n=1)$ occurs when the effects of heterogeneity and hydrophobicity compensate each other.

\subsection{Choice of model parameters.}

Specific Donnan volume, $V_{\mathrm{D}}$. For HS samples for which ion binding is well studied the parameter $b(-)$ of eq. (4) can be found in literature, see e.g. [5, 6]. This is also the case for PAHA, Saito et al. [45] used $b_{\mathrm{PAHA}}=0.63$. For the other HS investigated by Ishiguro et al. [22] no reliable specific information is available. Milne et al. [7] have discussed ion binding for a whole range of HS in relation to the NICA-Donnan model with eq. (4) for $V_{\mathrm{D}}$. By summarizing the results they have provided parameters for a generic $\mathrm{HA}$ and FA and the suggested $b$ parameters are: $b_{g \mathrm{HA}}=0.49$ and $b_{\mathrm{gFA}}=0.57$. For HS for which no specific data are available these values will be used as first estimates of the $b$ parameters.

The total salt concentration, $c_{\mathrm{s}}$, is obtained by adding the surfactant concentration to the background electrolyte concentration. Only for DPC close to the CMC the surfactant concentration contributes slightly to the salt concentration. Therefore, the constant background electrolyte concentration determines the salt concentration and the ionic activity coefficients are constant.

The maximum surfactant binding, $X^{\mathrm{m}}$. When it is assumed that, in principle, all acidic functional groups can bind a cationic surfactant ion $X^{\mathrm{m}}$ will be equal to the maximum 
draft IAP 2010; 3/6/2018

amount of proton charge. The latter can be obtained from literature when the proton binding isotherms have been fitted to the NICA-Donnan model or by using the titration method of Schnitzer and Gupta [46] to obtain the total number of acidic functional groups. As long as the surfactant binding is considerably smaller than $X^{\mathrm{m}}$ the model results are not very sensitive to the value of $X^{\mathrm{m}}$.

The initial amount of proton charge $X_{\mathrm{H}, 0}$ can best be obtained from the charge $-\mathrm{pH}$ curves of the HS. For PAHA the data of Saito et al. [45] can be used. When no reliable proton titration data are available $X_{\mathrm{H}, 0}$ can be estimated from titrations of the HS with the cationic polyelectrolyte, polyDADMAC, till the iso-electric point (IEP) of the complex is reached $[12,13]$. For DHA, IHA, SFA and LFA these data are available [22]. The maximum increment of the proton charge due to surfactant binding, $\Delta^{\mathrm{m}} X_{\mathrm{H}}$, can be estimated when the proton charge in the absence of surfactant and that at a given surfactant binding are known. For the latter proton charge at the IEP, $X_{\mathrm{H}, \mathrm{IEP}}$, of the surfactant-HS complex can be used. This quantity can be obtained from titrations of the HS with surfactant till the IEP of the complex is reached, see Ishiguro et al. [22]; both for CPC and DPC the values measured for CPC have been used, because the authors discuss that these are more reliable than those for DPC. From the available information $\Delta^{\mathrm{m}} X_{\mathrm{H}}$ can be obtained as $\left(X_{\mathrm{H}, \mathrm{IEP}}-X_{\mathrm{H}, 0}\right) / \theta_{\mathrm{S}, \mathrm{IEP}}$ with $\theta_{\mathrm{S}, \mathrm{IEP}}$ the degree of surfactant binding at the IEP of the HS-surfactant complex. As $X_{\mathrm{H}, \mathrm{IEP}}=X_{\mathrm{S} \text {,IEP }}$ this information can be used also for a comparison of the calculated and the experimental amount of surfactant bound at the IEP. With above estimates of the parameters the Donnan potentials can be calculated and the surfactant master curve, $X_{\mathrm{S}}$ vs. $c^{*} \mathrm{D}$, can be calculated. By fitting the surfactant master curve to the LFH equation (with $c=c^{*}$ D) the parameters $K^{*}$ and $n$ are obtained. Therefore, in the optimal situation the only fitting parameters of the present model are $K^{*}$ and $n$. 
draft IAP 2010; 3/6/2018

With the values of $K^{*}$ and $n$ and the other parameters the surfactant isotherms can be recalculated and predictions can be made for other solution conditions.

\section{Results and Discussion}

\subsection{Binding of CPC to PAHA: master curve and modeling of the isotherms.}

For the binding of CPC to PAHA accurate data at about constant $\mathrm{pH}$ of 5 and two salt concentrations, $0.005 \mathrm{~mol} / \mathrm{L}$ and $0.1 \mathrm{~mol} / \mathrm{L}$, are available [22]. These results are well suited to test the surfactant MC procedure using the adopted Donnan model. The parameters of the Donnan model are derived from the PAHA literature [22, 45] and collected in Table 1. The first step is the calculation of the Donnan potentials for each measured data point. With these potentials the $\mathrm{CP}^{+}$concentration in the Donnan phase is calculated and the isotherms can be re-plotted as binding vs. $c_{\mathrm{CP}+\mathrm{D}}$. The original $\mathrm{CPC}$ isotherms and the CPC-MC are depicted in Fig.1; panel (a) is a normal plot of the binding vs. $\log c$, panel (b) is a double logarithmic plot to clearly show the low surfactant concentration range. Given the fact that the parameters of the Donnan model (derived from proton binding modeling) are used without any further fitting, it may be concluded that the merging of the two surfactant isotherms into one MC is quite satisfactory up to the IEP. Note that around the IEP the experimental error in the measured isotherms is substantial because the binding values are obtained as a small difference of two large values (sample - blank).

Knowing that the Donnan model is adequate, the MC isotherm can be modeled with the LFH model by fitting $K^{*}$ and $n$. The calculated CPC model isotherms at the two salt concentrations follow by combining the LFH model with these parameters and the known Donnan model (together the LFH-D model). The results of the calculations are depicted 
draft IAP 2010; 3/6/2018

in Fig. 1 by the solid lines; the fitted intrinsic parameters are collected in Table 1. With these parameters the isotherms for both salt concentrations are well described by the model from very low surfactant concentrations till roughly the concentration of the IEP. The pseudo plateau in the binding isotherms close to the IEP, at binding values far below the maximum binding, is nicely predicted. The calculated LFH MC and LFH-D binding isotherms have quite different shapes due to the fact that the electrostatic interactions are "filtered-out" in the LFH MC. For surfactant binding values around the IEP of the CPCPAHA particles the calculated MC and bindings are very sensitive to the value of the proton charge at the IEP ( the latter affect the proton charge adjustment parameter, $\Delta^{\mathrm{m}} X_{\mathrm{H}}$ ). Changing the proton charge at the IEP at $0.005 \mathrm{~mol} / \mathrm{L}$ salt from $2.05 \mathrm{~mol} / \mathrm{kg}$ to 1.90 $\mathrm{mol} / \mathrm{kg}$ improves both the merging of the MC curves and the fit of the $0.005 \mathrm{~mol} / \mathrm{L}$ model isotherm around the IEP. Furthermore, it should be noted again that the experimental error in the binding values increases with increasing surfactant concentration; therefore, the IEP has been used also to determine the optimal position of the calibration line for the surfactant concentration [22]. Given these experimental uncertainties it may be concluded that the LFH-D model works well for the CPC-PAHA system. For PAHA the Donnan model as tested for CPC applies also for DPC. For DHA and IHA the Donnan model cannot be tested using the MC approach because no data are available at different salt concentrations. Here the only available criterion is that the shape of the MC should be logical.

\subsection{Modeling of DPC binding to PAHA, DHA and IHA.}

The Donnan model parameters for PAHA and DPC and that of PAHA and CPC at $0.005 \mathrm{~mol} / \mathrm{L}$ are the identical. For DHA and IHA the specific Donnan volume is 
draft IAP 2010; 3/6/2018

calculated with the generic $b$ parameter for HA [7]. The total acidity of DHA and IHA (as measured by the method of Martin et al. modified by Tsutsuki and Kuwatsuka) is obtained by Watanabe et al. [47]. The other proton charge values are derived from Table 1 in [22]. The isotherms of DPC to PAHA, DHA and IHA at pH about 5 and $0.005 \mathrm{~mol} / \mathrm{L}$ 1-1 background electrolyte are depicted in Figs. 2 to 4, respectively, both as double and single logarithmic plot. Next to the isotherm also the MC and its LFH fit plus the LFH-D description of the original isotherm are depicted. The fitted parameters $K^{*}$ and $n$ for each HA are collected in Table 1 together with the parameters of the Donnan model. The LFHD model description of the experimental data is quite good up to binding values close to the IEP. As expressed before, beyond the IEP the experimental error in the data increases steeply and the isotherms show a progressive binding that is at least partly caused by HA enhanced micellization. In the case of DPC this micellization could be micellization in solution enhanced by free HA and/or micellization enhanced by the HA-surfactant complex. Both forms of micellization are not included in the LFH-D model.

\subsection{Comparison of the LFH model parameters for CPC and DPC and for the different} $H A s$

The standard Gibbs energy of binding, $\Delta G^{*}$, between surfactant and HA can be evaluated using the $\ln K^{*}=\Delta G^{*} / R T$ values. For the CPC binding to PAHA $\ln K^{*}=11.41$ and for DPC binding this is 6.11; the energy difference is $5.30 R T$. This difference is due to the longer aliphatic chain of CPC than DPC, which leads to a stronger hydrophobicity of CPC than DPC. Per $\mathrm{CH}_{2}$ segment the difference is 5.30/4 = $1.32 k T$. This energy per $\mathrm{CH}_{2}$ segment compares well with the $1.1 k T$ that is obtained when the aliphatic chains are transferred from the solution to the core of CPC or DPC micelles. The fact that the 
draft IAP 2010; 3/6/2018

present value is higher than $1.1 k T$ is due to the fact that it is corrected for electrostatic interactions, whereas the $1.1 \mathrm{kT}$ is related to the transfer to a micelle that electrostatically repels the surfactant molecules.

A further comparison can be made for DPC binding to the three HAs. The observed $\ln K^{*}$ sequence is 6.11 for PAHA, 4.38 for IHA and 4.25 for DHA; therefore, the hydrophobicity ranking of the three HAs is PAHA > DHA > IHA. The differences must be related to the origin of the HAs: PAHA is extracted from brown coal, whereas IHA and DHA are extracted from the soil humic substances. The $n$ values indicate whether the hydrophobic cooperativity or the heterogeneity dominates. For PAHA-CPC $n=1.23$, i.e., the hydrophobic effect dominates over the heterogeneity effect while for PAHA-DPC with $n=0.78$ the heterogeneity strongly dominates. For DPC binding to DHA $n=0.95$ and for IHA $n=0.95$; in both cases the effect of heterogeneity on the affinity is nearly balanced by that of the hydrophobicity. These results also indicate the similarity of the soil HAs, IHA and DHA, and their difference with PAHA from brown coal.

\subsection{Prediction of DPC binding to PAHA at $0.025 \mathrm{~mol} / \mathrm{L}$ electrolyte and three $\mathrm{pH}$ values.}

To further test the quality of the present model the binding of DPC to PAHA at 0.025 mol/L background electrolyte at $\mathrm{pH} 4.5, \mathrm{pH} 7$ and $\mathrm{pH} 10$ is predicted using the LFH-D model with the parameters obtained above for the DPC-PAHA system. The LFH part of the model is exactly the same as above; the new impute is related to the Donnan part of the model: the new salt concentration determines the specific Donnan volume; the $\mathrm{pH}$ values determine the proton charge amounts; the amount of charge at the IEP is assumed to be $0.2 \mathrm{~mol} / \mathrm{kg}$ higher than $X_{\mathrm{H}, 0}$. The parameters are also summarized in Table 1 . The predictions can be compared with results of the DPC binding studies by Koopal et al. [29]. 
draft IAP 2010; 3/6/2018

However, these results are less accurate than those of Ishiguro et al. [22] because (1) the quality of the commercial surfactant electrodes that have been used was only moderate (low slopes of the calibration lines) and (2) no calibration of the electrochemical cell was carried out at the low equilibrium surfactant concentrations encountered with the isotherms. The calculated MCs are depicted in Fig. 5 and compared with the predicted MC (=LFH) of the DPC-PAHA system; the predicted (LFH-D) and the measured isotherms are depicted in Fig. 6. The symbols denote the experimental results, the curves the predictions. Considering that there is some uncertainty in the experimental $\mathrm{pH}$ values and that systematic differences will exist between the experimental results of Ishiguro et al. [22] and Koopal et al. [29] the differences between the LFH-MC and the experimental MCs (Fig. 5) are acceptable up to $\mathrm{pH} 7$, but for $\mathrm{pH} 10$ the experimental $\mathrm{MC}$ is steeper than the LFH curve. The model parameters (derived at $\mathrm{pH}$ 5) seem to underestimate the surfactant-surfactant interaction at $\mathrm{pH} 10$, which would imply that the increase of hydrophobicity by the large amount of bound surfactant is underestimated. The agreement between predicted and experimental pseudo plateau binding values near the IEP (Fig. 6) is good. Both model and experiment indicate that the number of acid groups that are dissociated mainly determines the pseudo-plateau of the surfactant adsorption. At low surfactant concentrations the predictions deviate seriously from the experimental results. This is either due to experimental inaccuracies at these low concentrations or to a shortcoming of the model. At the moment our conclusion is that the possibilities of the present LFH-D model to predict surfactant binding to HA outside the model calibration range are reasonably good. For a final decision about the quality of the model a more consistent set of experimental data at different $\mathrm{pH}$ values is required. 


\subsection{Modeling DPC binding to SFA and LFA}

The binding of DPC to SFA and LFA at $\mathrm{pH} 5$ and $0.005 \mathrm{~mol} / \mathrm{L}$ background electrolyte has also been analyzed. The maximum surfactant binding is assumed to be equal to the total number of acid groups for which the generic value for FAs is used [7]. The other parameters are derived from Table 1 in [22]. The parameter values are collected in Table 1. For the calculation of the specific Donnan volume initially Milne's generic $b$ parameter for FAs (0.57) was used. However, the resulting MCs were far from satisfactory around the IEP. To obtain for both FAs a MC with a logical shape around the IEP the value of $b$ had to be raised to 0.9 ; this value leads to rather large specific Donnan volumes, but the initial Donnan potentials of about -40 to $-50 \mathrm{mV}$ are of the right order of magnitude. The MCs obtained with $b=0.9$ and the original isotherms are depicted in Fig. 7 and 8 for, respectively, LFA and SFA.

Fitting the LFH equation to the MC up to the IEP was for both FAs not very satisfactory: at adsorbed amounts of about $0.5 \mathrm{~mol} / \mathrm{kg}$ the steep rise of the MC cannot be followed by the $\mathrm{LFH}$ equation. The parameters $n$ and $K^{*}$ used to obtain the LFH isotherms depicted in Figs. 7 and 8 are collected in Table 1. A similar weakness occurs with the isotherms calculated with the LFH-D model. The poor description is due to the fact that the heterogeneity of the FAs is dominant $(n<$ 1) and the initial affinity of DPC for FAs is fairly low. Due to DPC binding the FA-DPC particles become hydrophobic and at a DPC binding of about $0.5 \mathrm{~mol} / \mathrm{kg}$ an additional binding cooperativity occurs and/or some enhanced micellization is induced by the FA-DPC particles. This new cooperativity effect is not included in the LFH model. A better description is to be expected from a modified Gu-model for surfactant binding. In the $\mathrm{Gu}$ type model the first adsorbing surfactant molecules are considered as nuclei for cooperative binding at higher bound amounts [48]. The modification of the Gu model to heterogeneous binding sites and the incorporation of electrostatics remains a task for the future. The stronger cooperativity effect with surfactant binding to FA than to HA has been found before by Yee et al. [21]. They could model the surfactant binding to HAs with the Langmuir equation, but needed the Hill equation for 
draft IAP 2010; 3/6/2018

surfactant binding to FA. Note that in Yee's way of modeling three effects determine the nonideality parameter: the HS heterogeneity (as in the Langmuir-Freundlich equation), the pseudo heterogeneity due to the diminishing electrostatic attraction (as in the modified HendersonHasselbalch equation [49]) and the cooperativity (as in the Hill equation).

\section{Conclusions}

A predictive model has been developed for surfactant binding. The model consists of a Donnan (D) part that quantifies the electrostatic interactions and a LangmuirFreundlich-Hill (LFH) part that quantifies specific interactions. The LFH part takes both heterogeneity and hydrophobic effects into account. The model is analogous to the NICA-Donnan model for proton and metal ion binding to HS, but competition of the surfactant cations with the protons is neglected.

The LFH-D model describes the binding of cationic surfactants to HAs very well from extremely low surfactant binding up to about the IEP of the particles loaded with surfactant. With the calibrated LFH-D model predictions of surfactant binding to HAs can be made at conditions outside the calibration range. For FAs the LFH-D model is only suited to describe the binding from very low adsorbed amounts till adsorbed amounts of about $0.5 \mathrm{~mol} / \mathrm{kg}$. Beyond $0.5 \mathrm{~mol} / \mathrm{kg}$ the surfactant binding rises more steeply than predicted with the model due hydrophobicity induced by the already adsorbed surfactant molecules. This aspect is not included in the LFH model.

\section{Acknowledgements}

We thank Dr. Takumi Saito of the University of Tokyo for his help with the calculations of the Donnan potential. 


\section{References}

[1] J.C.M. De Wit, W.H. Van Riemsdijk, M.M. Nederlof, D.G. Kinniburgh, L.K. Koopal, Analysis of ion binding on humic substances and the determination of intrinsic affinity distributions, Anal. Chim. Acta 232 (1990) 189-207.

[2] M.M. Nederlof, W.H. Van Riemsdijk, L.K. Koopal, Comparison of semianalytical methods to analyze complexation with heterogeneous ligands, Environ. Sci. Technol. 26 (1992) 763-771.

[3] P. Merdy, S. Huclier, L. K. Koopal, Modeling metal-particle interactions with an emphasis on natural organic matter, Environ. Sci. Technol. 40 (2006) 7459-7466.

[4] L.K. Koopal, W.H. Van Riemsdijk, J.C.M. De Wit, M.F. Benedetti, Analytical isotherm equations for multicomponent adsorption to heterogeneous surfaces, J. Colloid Interface Sci. 166 (1994) 51-60.

[5] D.G. Kinniburgh, W.H. van Riemsdijk, L.K. Koopal, M. Borkovec, M.F. Benedetti, M.J. Avena, Ion binding to natural organic matter: competition, heterogeneity, stoichiometry and thermodynamic consistency, Colloids Surf. A 151 (1999) 147-166.

[6] M. F. Benedetti, C. J. Milne, D. G. Kinniburgh, W. H. Van Riemsdijk, L. K. Koopal, Metal-ion binding to humic substances - Application of the nonidel competitive adsorption model, Environ. Sci. Technol. 29 (1995) 446-457.

[7] C. J. Milne, D. G. Kinniburgh, W. H. van Riemsdijk, E. Tipping, Generic NICADonnan model parameters for metal-ion binding by humic substances, Environ. Sci. Technol. 37 (2003) 958-971.

[8] L. K. Koopal, W. H. van Riemsdijk, D. G. Kinniburgh, Humic matter and contaminants. General aspects and modeling metal ion binding, Pure Appl. Chem. 73 (2001) 2005-2016.

[9] L. K. Koopal, T. Saito, J. P. Pinheiro, W. H. Van Riemsdijk, Ion binding to natural organic matter: General considerations and the NICA-Donnan model, Colloids Surf. A 265 (2005) 40-54.

[10] W.H . Van Riemsdijk, L.K. Koopal, D.G. Kinniburgh, M.F. Benedetti, L. Weng, Modeling the interactions between humics, ions, and mineral surfaces, Environ. Sci. Technol. 40 (2006) 7473-7480.

[11] E. Tipping, Humic ion-binding model VI: An improved description of the interactions of protons and metal ions with humic substances, Aquat. Geochem. 4 (1998) 3-48.

[12] S.-K. Kam, J. Gregory, The interaction of humic substances with cationic polyelectrolytes, Water Res. 35 (2001) 3557-3566.

[13] W.F. Tan, W. Norde, L.K. Koopal, Charge determination of dissolved humic substances by colloid titration with a flexible cationic polyelectrolyte, Environ. Sci. Technol. Submitted

[14] B. Bolto, G. Abbt-Braun, D. Dixon, R. Eldridge, F. Frimmel, S. Hesse, S. King, M. Toifl, Experimental evaluation of cationic polyelectrolytes for removing natural organic matter from water, Water Sci. Technol. 40 (1999) 71-79.

[15] W. F. Tan, L. K. Koopal, L. P. Weng, W. H. van Riemsdijk, W. Norde, Humic acid protein complexation, Geochim. Cosmochim. Acta 72 (2008) 2090-2099.

[16] W. F. Tan, L. K. Koopal, W. Norde, Interaction between Humic Acid and Lysozyme, Studied by Dynamic Light Scattering and Isothermal Titration Calorimetry, Environ. Sci. Technol. 43 (2009) 591-596. 
[17] J.H.A. Butler, J.N. Ladd, Importance of the molecular weight of humic and fulvic acids in determining their effects on protease activity, Soil Biol Biochem. 3 (1971) 249-257.

[18] C.T. Chiou, D.E. Kile, T.I. Brinton, R.L. Malcolm, J.A. Leenheer, P. Maccarth, A comparison of water solubility enhancements of organic solutes by aquatic humic materials and commercial humic acids, Environ. Sci. Technol. 21 (1987) 1231-1234.

[19] B. Xing, J. Pignatello, Dual-mode sorption of low-polarity compounds in glassy poly(vinyl chloride) and soil organic matter, Environ. Sci. Technol. 31 (1997) 792799.

[20] Y.H. Yang, L.K. Koopal, Immobilisation of humic acids and binding of nitrophenol to immobilised humics, Colloids Surf. A 151 (1999) 201-212.

[21] M.M. Yee, T. Miyajima, N. Takisawa, Evaluation of amphiphilic properties of fulvic acid and humic acid by alkylpyridinium binding study, Colloids surfaces A 272 (2006) 182-188.

[22] M. Ishiguro, W. F. Tan, L. K. Koopal, Binding of cationic surfactants to humic substances, Colloids Surf. A 306 (2007) 29-39.

[23] S. Sakashita, Electron microscopic study of liver tissue after cutaneous administration of detergents J. Clin. Electron Microscopy 12 (1979) 189-216.

[24] P. Somasundaran, D. W. Fuerstenau, Mechanisms of Alkyl Sulfonate Adsorption at the Alumina-Water Interface, J. Phys. Chem. 70 (1966) 90-96.

[25] M.R. Böhmer, L.K. Koopal, Adsorption of ionic surfactants on variable-charge surfaces, 1. Charge effects and structure of the adsorbed layer, Langmuir 8 (1992) 2649-2659.

[26] E.M. Lee, L.K. Koopal, Adsorption of cationic and anionic surfactants on metal oxide surfaces: Surface charge adjustment and competition effects, J. Colloid Interface Sci. 177 (1996) 478-489.

[27] T.P. Goloub, L.K. Koopal, Adsorption of cationic surfactants on silica. Comparison of experiment and theory, Langmuir 13 (1997) 673-681.

[28] L.K. Koopal, Modeling association and adsorption of surfactants, in: K. Esumi, M. Ueno (Eds.), Structure-performance relationships in surfactants, Second edition, Surfactant Science Series vol. 112, Marcel Dekker, New York etc., 2003, pp. 111-196.

[29] L.K. Koopal, T.P. Goloub, T.A. Davis, Binding of ionic surfactants to purified humic acid, J. Colloid Interf. Sci. 275 (2004) 360-367.

[30] M. Matsuda, A. Kaminaga, K. Hayakawa, N. Takisawa, T. Miyajima, Surfactant binding by humic acids in the presence of divalent metal salts, Colloids Surfs. A 347 (2009) 45-49.

[31] J.C.M. De Wit, W.H. Van Riemsdijk, L.K. Koopal, Proton binding to humic substances, 1. Electrostatic effects, Environ. Sci. Technol. 27 (1993) 2005-2014.

[32] B.M. Bartschat, S.E. Cabaniss, F.M.M. Morel, Oligoelectrolyte model for cation binding by humic substances, Environ. Sci. Technol. 26 (1992) 284-294.

[33] M.F. Benedetti, W.H. Van Riemsdijk, L.K. Koopal, Humic substances considered as a heterogeneous donnan gel phase, Environ. Sci. Technol. 30 (1996) 1805-1813.

[34] N.D. Bryan, N. Hesketh, F.R. Livens, E. Tipping, M.N. Jones, Metal ion-humic substance interaction - A thermodynamic study, J. Chem. Soc.-Faraday Trans. 94 (1998) 95-100.

[35] E. Tipping, Humic ion-binding model VI: An improved description of the interactions of protons and metal ions with humic substances, Aquat. Geochem. 4 (1998) 3-48. 
[36] N.D. Bryan, D.M. Jones, M. Appleton, F.R. Livens, M.N. Jones, P. Warwick, S. King, A. Hall, A physicochemical model of metal-humate interactions, PCCP Phys. Chem. Chem. Phys. 2 (2000) 1291-1300.

[37] M.J. Avena, L.K. Koopal, W.H. Van Riemsdijk, Proton binding to humic acids: Electrostatic and intrinsic interactions, J. Colloid Interface Sci. 217 (1999) 37-48.

[38] T. Saito, S. Nagasaki, S.Tanaka, L. K. Koopal, Electrostatic interaction models for ion binding to humic substances, Colloids and Surfaces A 265 (2005) 104-113.

[39] J.T. Davies, E.K. Rideal, Chapter 2, in; J.T. Davies (Ed.), Interfacial Phenomena, Academic Press, New York, London, 1961.

[40] E. Companys, J. Llu Garces , J. Salvador, J.Galceran, J. Puy, F. Mas, Electrostatic and specific binding to macromolecular ligands - A general analytical expression for the Donnan volume, Colloids Surfaces A 306 (2007) 2-13.

[41] A.V. Hill, A new mathematical treatment of changes of ionic concentration in muscle and nerve under the action of electric currents, with a theory as to their mode of excitation, J. Physiol. (Lond.) 40 (1910) 190-224.

[42]K. A. Dill, S. Bromberg, Molecular Driving Forces: Statistical Thermodynamics in Chemistry and Biology, Garland Science, New York, 2003.

[43]R. J. Sips, On the Structure of a Catalyst Surface, J. Chem. Phys. 16 (1948) 490-495.

[44]M. Jaroniec, Physical adsorption on heterogeneous solids, Adv. Colloid Interface Sci. 18 (1983) 149-225.

[45] Saito, T., L. K. Koopal, W. H. van Riemsdijk, S. Nagasaki, S. Tanaka, Adsorption of humic acid on goethite: Isotherms, charge adjustments, and potential profiles, Langmuir, 20 (2004) 689-700.

[46] M. Schnitzer, U.C. Gupta, Determination of Acidity in Soil Organic Matter, Soil Sci. Soc. Am. Proc. 29 (1965) 274-277.

[47] A. Watanabe, K. Itoh, S. Arai, S. Kuwatsuka, Comparison of the composition of humic and fulvic-acids prepared by the IHSS method and Nagoya method, Soil Sci. Plant Nutri. 40 (1994) 601-608.

[48] B.-Y. Zhu, T. Gu, Surfactant adsorption at solid liquid interfaces, Adv. Colloid Interface Sci. 37 (1991) 1-32.

[49] M. Mandel, The potentiometric titration of weak polyacids, Eur. Polym. J. 6 (1970) 807-822. 


\section{Figure legends}

Fig. 1. CPC binding to PAHA: experimental isotherms at 0.005 and $0.1 \mathrm{~mol} / \mathrm{L} 1-1$ salt, CPC-PAHA MC (Donnan model) and model fits to the MC and the individual isotherms. Panel (a) and (b) depict the same data in different ways, panel (b) gives emphasis to the low concentration range.

Fig. 2. DPC binding to PAHA at $\mathrm{pH}$ about 5 and $0.005 \mathrm{~mol} / \mathrm{L}$ salt; experimental results, the MC, the model fit to the MC and the calculated DPC binding isotherm. Panel (a) and (b) depict the same data in different ways, panel (b) gives emphasis to the low concentration range.

Fig. 3. DPC binding to DHA at $\mathrm{pH}$ about 5 and $0.005 \mathrm{~mol} / \mathrm{L}$ salt; experimental results, the MC, the model fit to the MC and the calculated DPC binding isotherm. Panel (a) and (b) depict the same data in different ways, panel (b) gives emphasis to the low concentration range.

Fig. 4. DPC binding to IHA at $\mathrm{pH}$ about 5 and $0.005 \mathrm{~mol} / \mathrm{L}$ salt; experimental results, the MC, the model fit to the MC and the calculated DPC binding isotherm. Panel (a) and (b) depict the same data in different ways, panel (b) gives emphasis to the low concentration range.

Fig. 5. Comparison of the predicted MC of the DPC-PAHA system with the calculated MCs for three $\mathrm{pH}$ values (4.5, 7 and 10) and a salt concentration of $0.025 \mathrm{~mol} / \mathrm{L}$.

Fig. 6. Predicted and measured binding isotherms for the DPC-PAHA system at 0.025 mol/L 1-1 background electrolyte and three $\mathrm{pH}$ values (4.5, 7 and 10).

Fig. 7. Analysis of the DPC binding to LFA at $\mathrm{pH} 5$ and $0.005 \mathrm{~mol} / \mathrm{L} 1-1$ background electrolyte. Solid symbols: experimental data; open symbols calculated MC. The drawn curves are the model fits to the MC and the experimental isotherm. Panel (a) and (b) depict the same data in different ways, panel (b) gives emphasis to the low concentration range.

Fig. 8. Analysis of the DPC binding to SFA at pH 5 and $0.005 \mathrm{~mol} / \mathrm{L} 1-1$ background electrolyte. Solid symbols: experimental data; open symbols calculated MC. The drawn curves are the model fits to the MC and the experimental isotherm. Panel (a) and (b) depict the same data in different ways, panel (b) gives emphasis to the low concentration range. 
Figures Ishiguro, Koopal
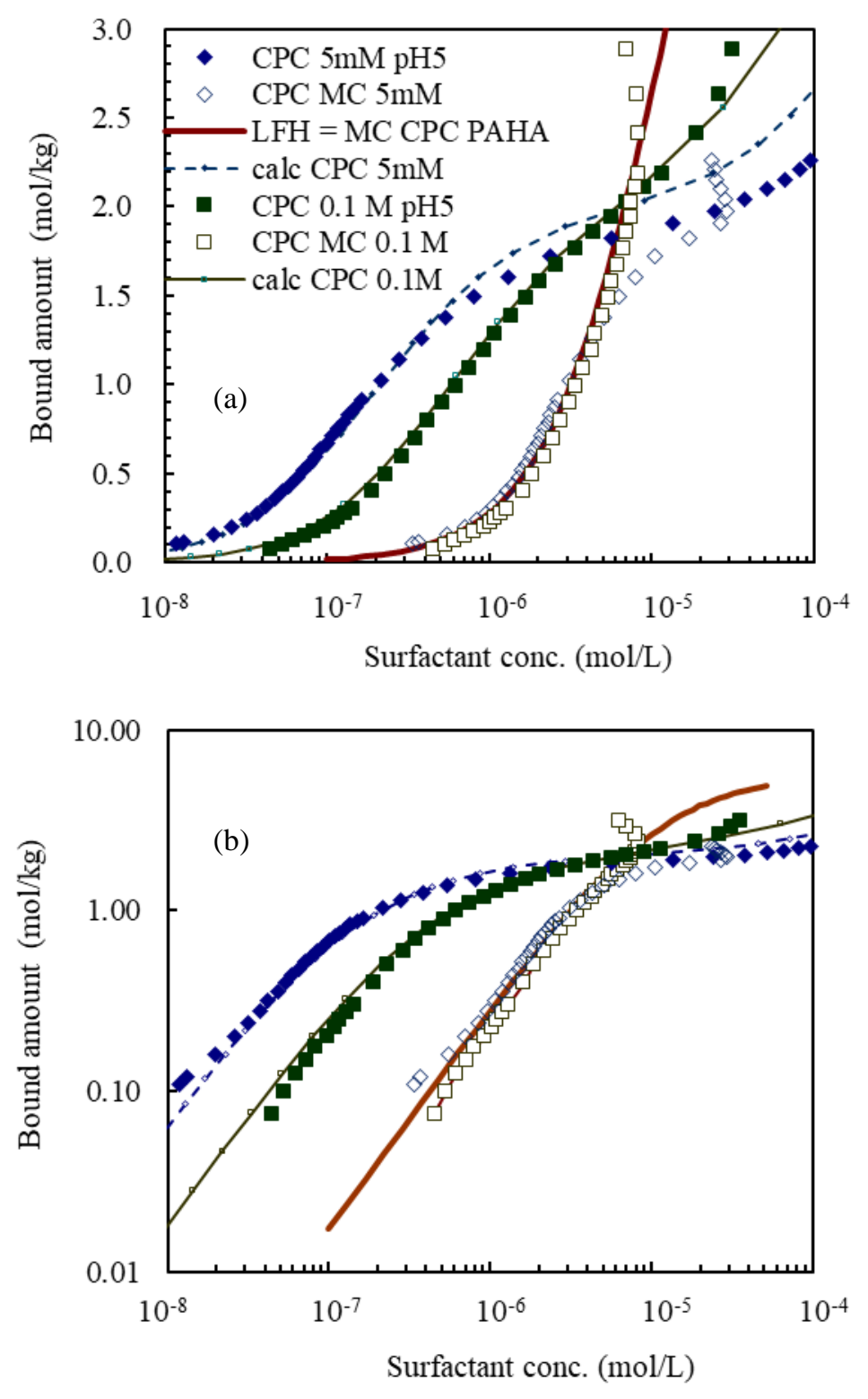

Fig. 1. CPC binding to PAHA: experimental isotherms at 0.005 and $0.1 \mathrm{~mol} / \mathrm{L} 1-1 \mathrm{salt}$, CPC-PAHA MC (Donnan model) and model fits to the MC and the individual isotherms. Panel (a) and (b) depict the same data in different ways, panel (b) gives emphasis to the low concentration range. 

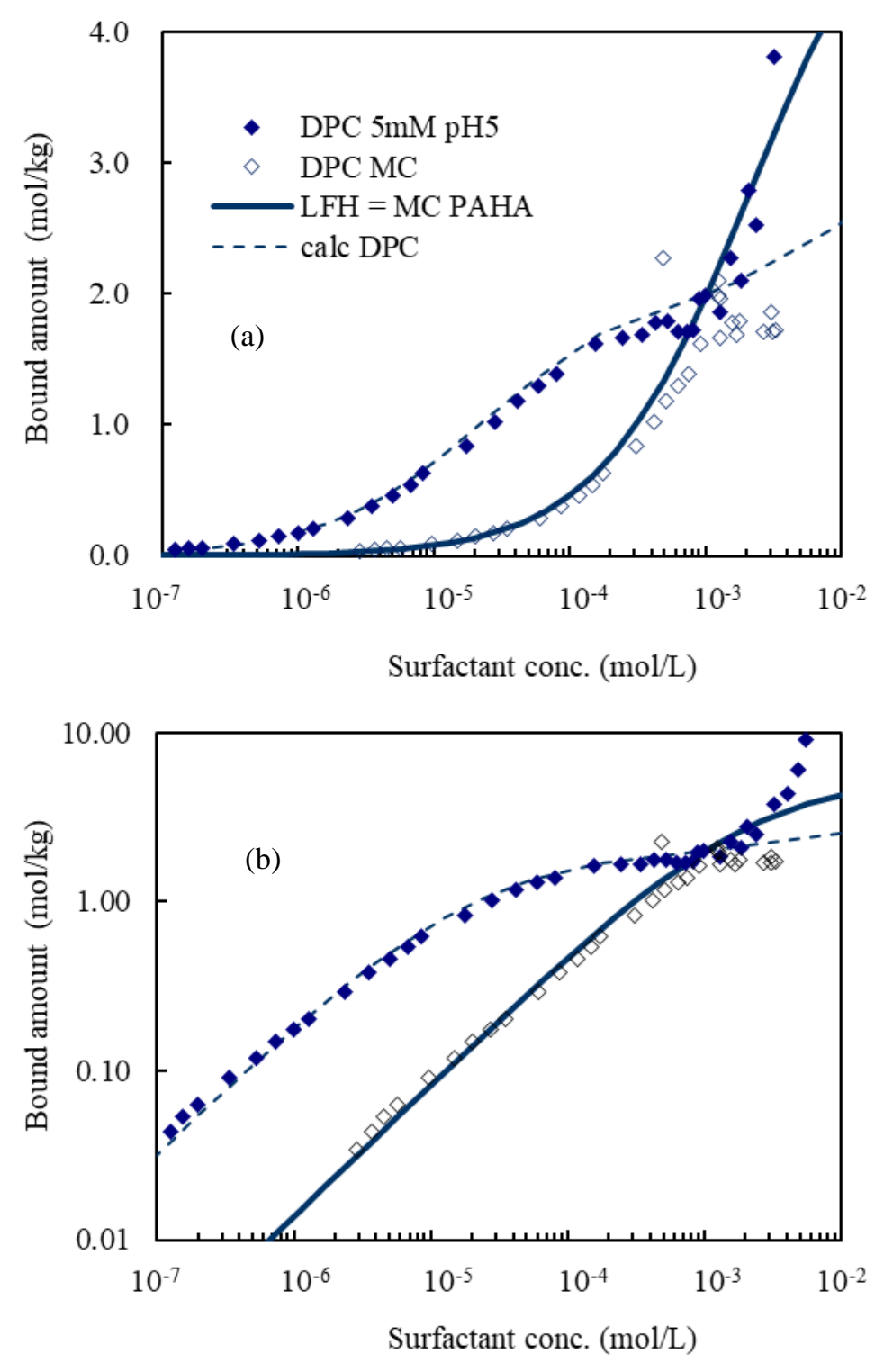

Fig. 2. DPC binding to PAHA at $\mathrm{pH}$ about 5 and $0.005 \mathrm{~mol} / \mathrm{L}$ salt; experimental results, the MC, the model fit to the MC and the calculated DPC binding isotherm. Panel (a) and (b) depict the same data in different ways, panel (b) gives emphasis to the low concentration range. 

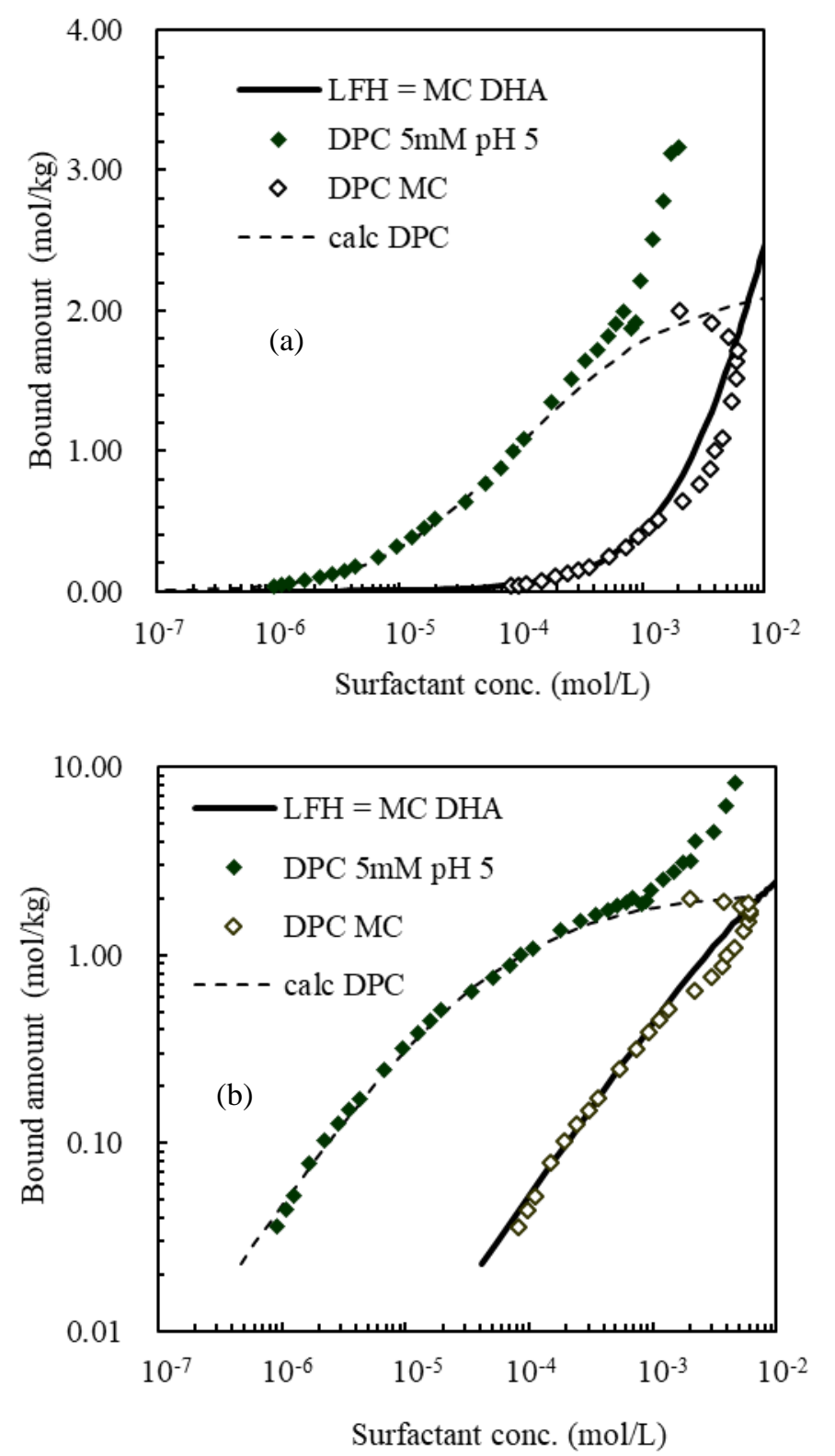

Fig. 3. DPC binding to DHA at $\mathrm{pH}$ about 5 and $0.005 \mathrm{~mol} / \mathrm{L}$ salt; experimental results, the MC, the model fit to the MC and the calculated DPC binding isotherm. Panel (a) and (b) depict the same data in different ways, panel (b) gives emphasis to the low concentration range. 

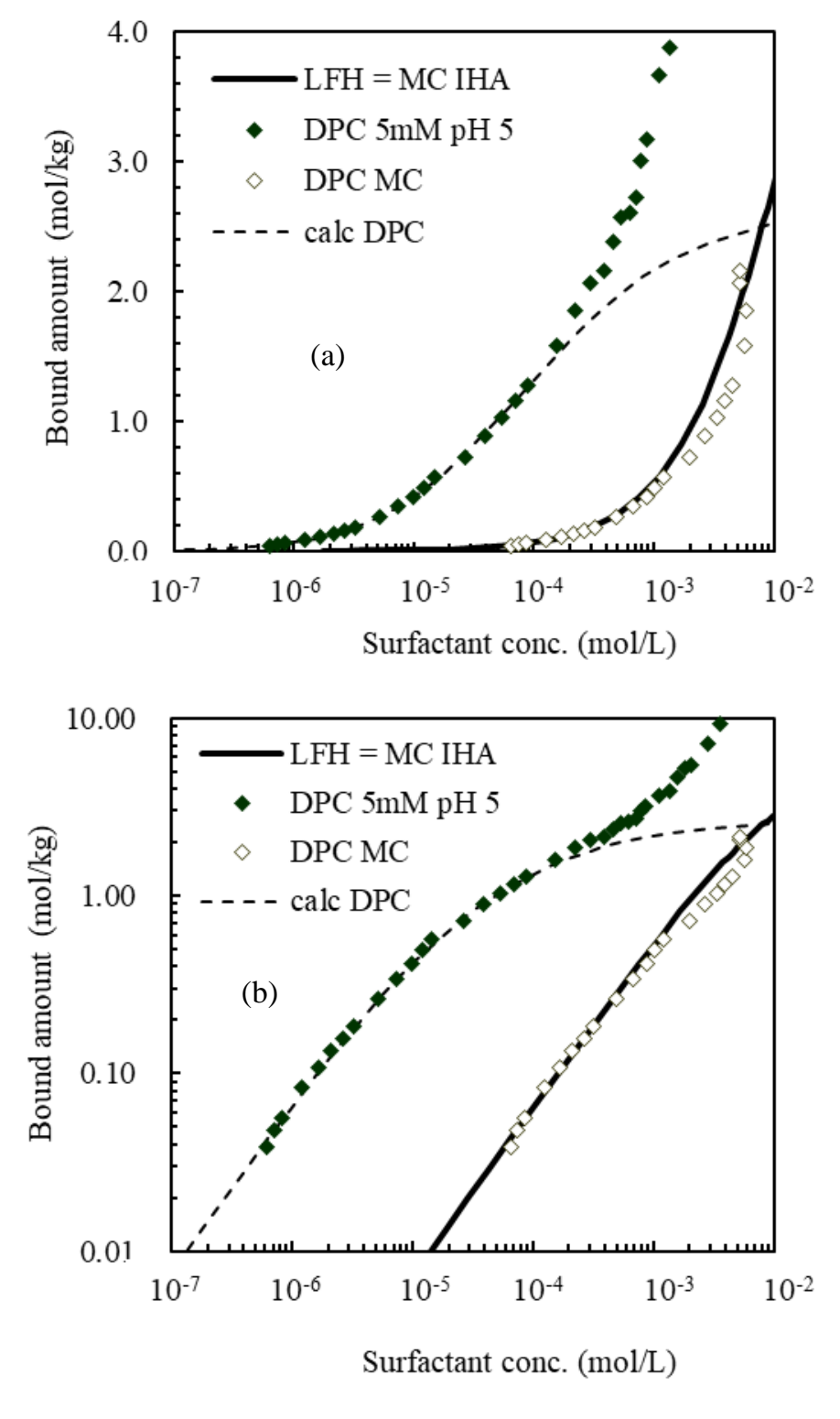

Fig. 4. DPC binding to IHA at $\mathrm{pH}$ about 5 and $0.005 \mathrm{~mol} / \mathrm{L}$ salt; experimental results, the $\mathrm{MC}$, the model fit to the MC and the calculated DPC binding isotherm. Panel (a) and (b) depict the same data in different ways, panel (b) gives emphasis to the low concentration range. 
draft IAP 2010; 3/6/2018

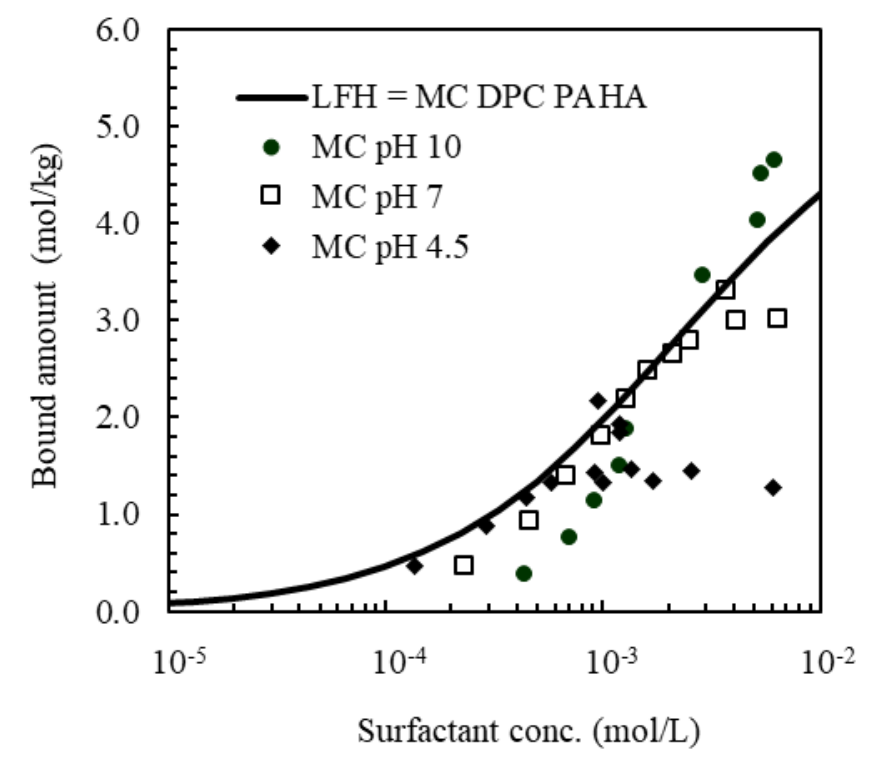

Fig. 5. Comparison of the predicted MC of the DPC-PAHA system with the calculated MCs for three $\mathrm{pH}$ values $(4.5,7$ and 10) and a salt concentration of $0.025 \mathrm{~mol} / \mathrm{L}$. 


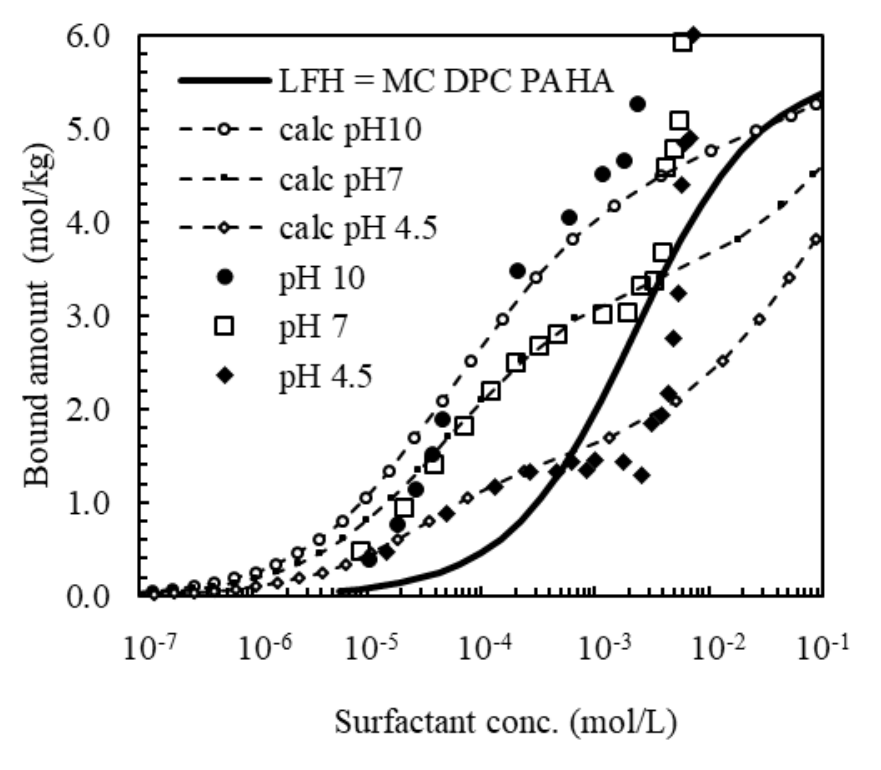

Fig. 6. Predicted and measured binding isotherms for the DPC-PAHA system at 0.025 mol/L 1-1 background electrolyte and three $\mathrm{pH}$ values $(4.5,7$ and 10$)$. 

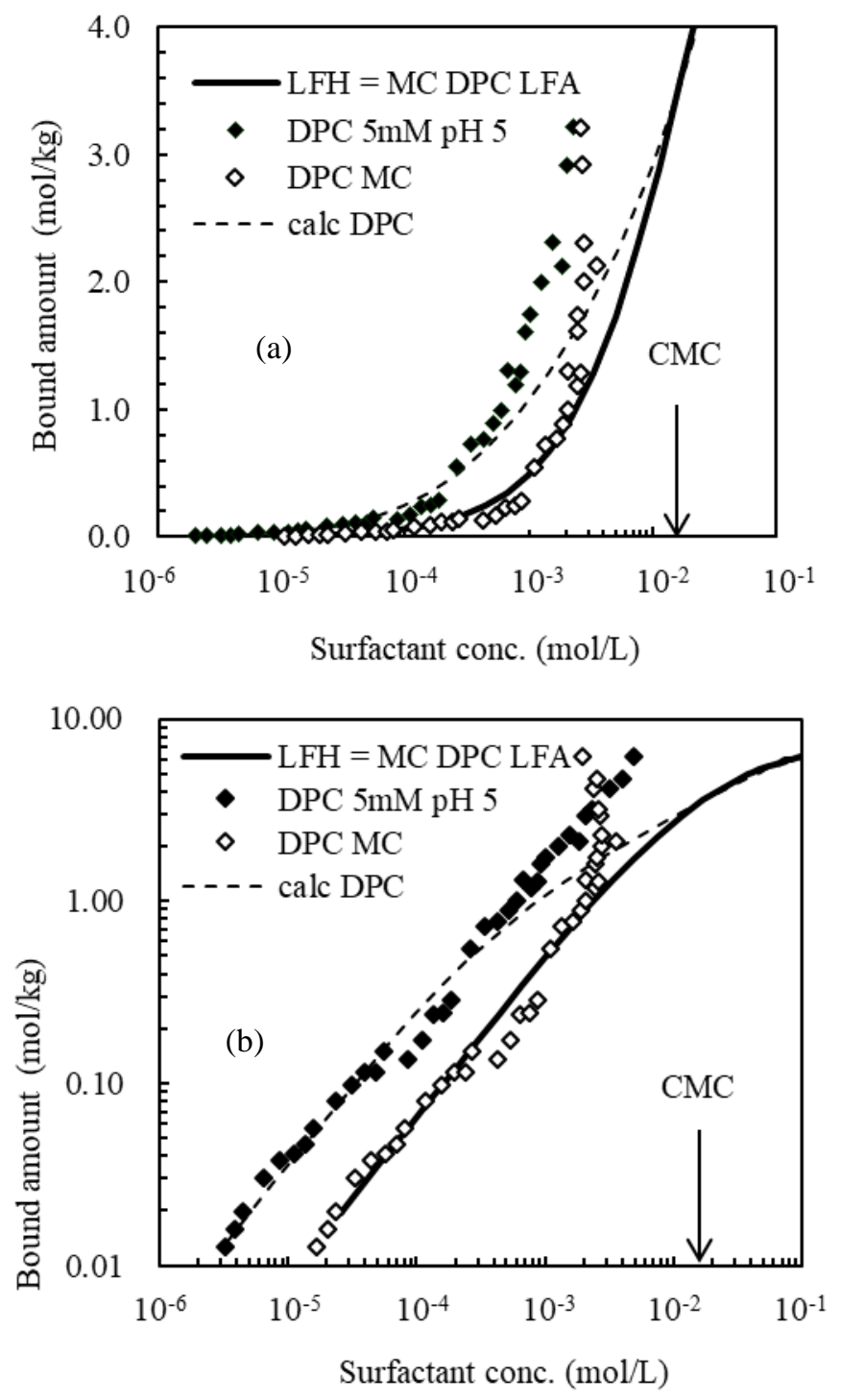

Fig. 7. Analysis of the DPC binding to LFA at $\mathrm{pH} 5$ and $0.005 \mathrm{~mol} / \mathrm{L} 1-1$ background electrolyte. Solid symbols: experimental data; open symbols calculated MC. The drawn curves are the model fits to the MC and the experimental isotherm. Panel (a) and (b) depict the same data in different ways, panel (b) gives emphasis to the low concentration range. 

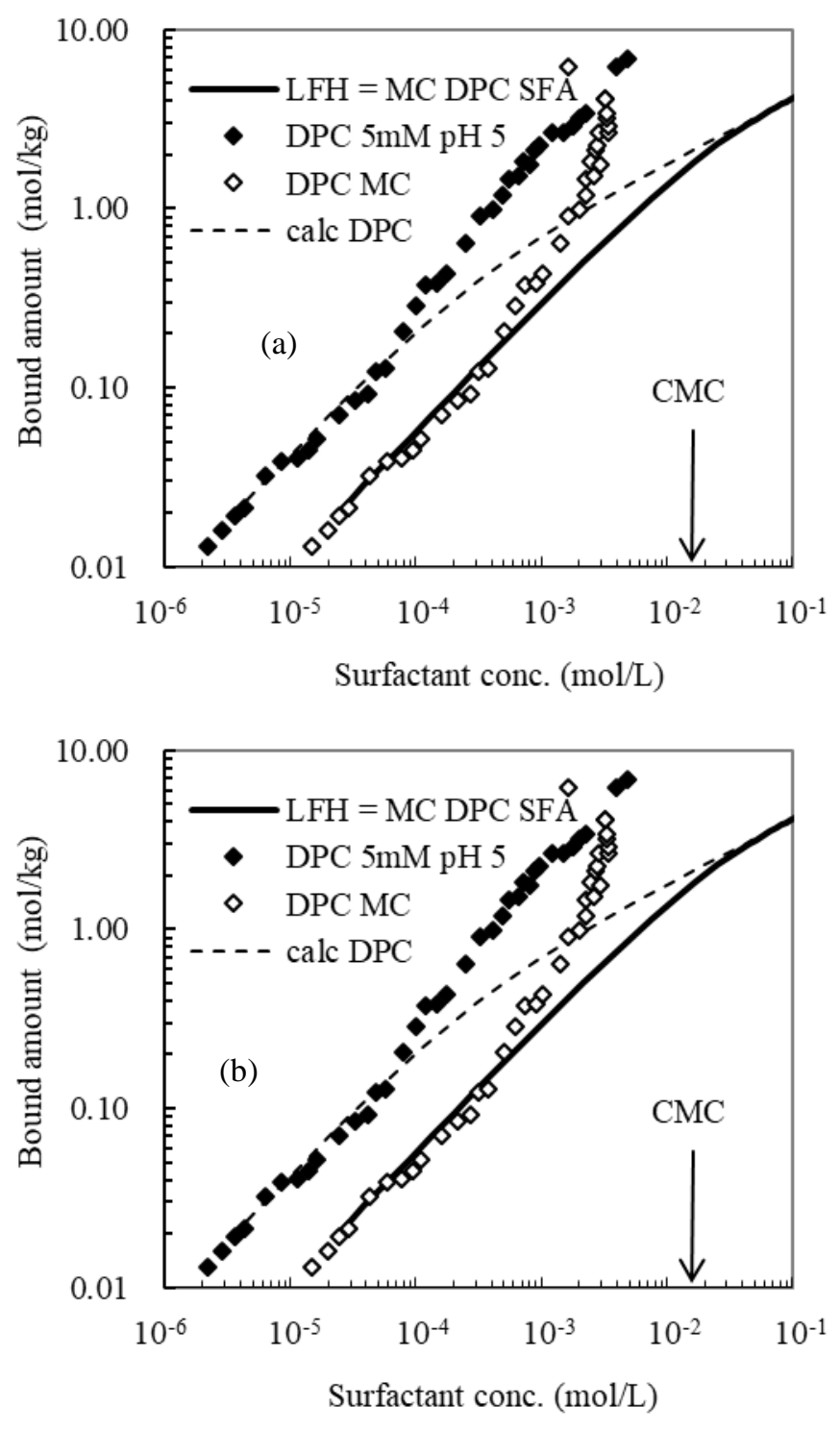

Fig. 8. Analysis of the DPC binding to SFA at $\mathrm{pH} 5$ and $0.005 \mathrm{~mol} / \mathrm{L} 1-1$ background electrolyte. Solid symbols: experimental data; open symbols calculated MC. The drawn curves are the model fits to the MC and the experimental isotherm. Panel (a) and (b) depict the same data in different ways, panel (b) gives emphasis to the low concentration range. 